\title{
The Evaluation of Fracture Energy at the Interface between Grilled Fish Protein and Metal
}

\author{
Naoko Honda ${ }^{1)^{*}}$, Noritsugu Umehara ${ }^{2)}$, Takayuki Tokoroyama ${ }^{2)}$, Ryusei Ohashi ${ }^{3)}$ and Akira Hanafusa ${ }^{3)}$ \\ ${ }^{1)}$ Nagoya Municipal Industrial Research Institute, Machine Systems Research Office \\ 41-4-3 Rokuban, Atsuta-ku, Nagoya, Aichi 456-0048, Japan \\ ${ }^{2)}$ Department of Mechanical Science and Engineering, Nagoya University \\ Furo-cho, Chikusa-ku, Nagoya, Aichi 464-8603, Japan \\ 3) Research \& Development Div., Rinnai Corporation \\ 2-1 Nishi Haccho, Akita, Oguchi, Niwa-gun, Aichi 480-0132, Japan \\ *Corresponding author: honda.naoko@nmiri.city.nagoya.jp
}

( Manuscript received 9 April 2009; accepted 4 February 2010; published 15 February 2010 )

\begin{abstract}
We have a problem of fish adhering to the metal grids of grill during grilling fish strongly. This problem is difficult to be solved because the grids of grill coated the fluorine resin which was used generally could be disintegrated at more than $300{ }^{\circ} \mathrm{C}$. This adhesion mechanism is so complicated phenomena. As a usual, the maximum peeling force to peel off fish from the grids is the parameter to evaluate the adhesion properties of grids. However, such maximum peeling force is not basic data with physical meaning because it depends on the sequence of peeling fish. Therefore we proposed a new evaluation method. We focused on the adhesion of fish protein against a rod specimen. We poured the fish protein on a plate specimen, put a rod specimen on them and heated them in an electric furnace. One minute later, we pulled up an end of the rod specimen in the constant speed. During this experiment, we measured the peeling force and the displacement of the end of the rod specimen. After this experiment, we observed the plate specimen with SEM and EDS to evaluate the contact area at the interface between the rod specimen and denatured fish protein.
\end{abstract}

Keywords: fish protein, adhesion, peeling force, the grids of grill

\section{Introduction}

During grilling fish, the fish adheres to the grids of grill. We have a still unsolved issue on the adhesion of the denatured fish body by heating. The fluorine resin that is coated on the grids of grill generally could be disintegrated at high temperature more than $300{ }^{\circ} \mathrm{C}$. Therefore we should find anti-stick surface treatment or material against the liquid exuded from fish during grilling fish which included protein, oil, water, and so on, we call the liquid fish protein, and this treatment or material has to be heat-endurance material. Furthermore, the conventional evaluation method to evaluate the adhesion properties of grids is to measure the maximum adhesion force by peeling off fish from the grids. The parameter was only the maximum adhesion force. But such the maximum adhesion force is not basic data with physical meaning because it depends on the sequence of peeling fish. Peeling does not occur for a moment. Therefore we should consider the crack propagation during peeing. However no researchers considered the fracture mechanics at the interface between a fish and grid rods.

In this study, we propose the new method to evaluate fracture energy at the interface between grilled fish and metal grid rods. And we discuss about the effectiveness and problems of this proposed method.

\section{New evaluation method of interface fracture energy}

During grilling fish, a large number of drops of fish protein were dropped down on the tray under the grids of grill. And we observed the fish and grill rods during grilling fish carefully, we thought that the strong adhesion between the fish and the grill rod caused the fish protein. The fish protein was exuded from the fish by heating and became charcoal around grill rods which contact the fish. This charcoal dominated the adhesion against the grill rod. So we focused on the interface between the fish protein and a rod specimen.

Here in order to overcome this problem, we proposed this new easy evaluation method. The evaluation method 
was to peel off between this fish protein and a rod specimen, which is $30 \mathrm{~mm}$ length cut of grids, for the measurement of fracture mechanics between them. This evaluation method has three advantages, firstly to measure the peeling force between the fish protein and a rod specimen easily without the fish and the gas cooker, secondly to get the basic and physical load-displacement curve with the experimental apparatus, and thirdly to judge easily whether the grill rod material is useful as the grill.

On the basis of fracture mechanics, the difference between external applied work $W$ and strain energy of a rod specimen $U$ equals to the interface fracture energy $\Gamma$ to create new fracture surfaces ${ }^{1,2)}$. Therefore, the condition of the onset of the fracture at the interface depends on the elastic properties and the shapes of the interface and the bulk materials. But, in case of the interface between a fish and a grill rod, the elastic property and the shape of the fish can not be estimated easily. Therefore, we used the fish protein to neglect the deformation of the fish body for the peeling experiment. And the fish protein would be brittle. Therefore, we thought that the interface fracture energy between the fish protein and the rod specimen dominated the adhesion between the fish and the grill rod.

In order to estimate the interface fracture energy $\Gamma$, we should evaluate the external applied work $W$ and the strain energy $U$ by the external applied work $W$ during the adhesion experiment. The external applied work $W$ was estimated by the measurement of adhesion force $F$ and the displacement $d$ at the pulling up point pulled up by the hook, which was at $2.5 \mathrm{~mm}$ from the end of the rod specimen as shown in Fig. 1. The shapes of the two specimens are shown in the Fig. 1. The rod specimen is fixed with the plate specimen by the fish protein as the cantilever. After heating the rod specimen was started to be pulling up to the end of the rod specimen as shown in Fig. 2 (a) and increased up to the perfect separation of the rod specimen from the plate specimen as shown in Fig. 2(b). During peeling process of the rod specimen, the adhesion force $F$ and the displacement $d$ of the end of the rod specimen were measured to estimate the external applied work $W$. After the adhesion experiment, the contact area $A$ was estimated by the observation. We can estimate the interface fracture energy $\Gamma$ by the following equation (1).

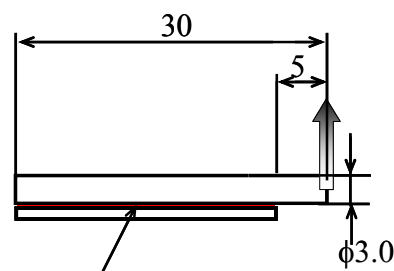

Fish protein

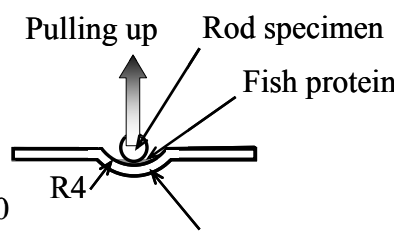

Plate specimen
Fig. 1 The schematic of a rod specimen and a plate specimen

$$
\Gamma=W-U
$$

And because the interface fracture energy $\Gamma$ did not consider the influence of the contact area, we used the interface fracture energy per unit contact area, $G$. And so $G$ can be expressed by the next equation (2) to normalize result of a measurement.

$$
G=\frac{d}{d A}(W-U)=\frac{d \Gamma}{d A}
$$

However, base material of specimens and fixtures are stainless steel (SUS304) and so the strain energy $U$ can ignore in comparison with the fish protein. Therefore, the strain energy $U$ is regarded as 0 .

\section{Experiment}

\subsection{Fish protein}

When we observed the adhesion behavior of fish against grilling rods, we believed that fish protein come from fish during grilling had a role of bond. During grilling fish, a large number of the fish protein as liquid drops from fish were dropped down on the tray under the grids of grill and we collected them. We investigated this fish protein. The ingredient of fish protein is $89.8 \%$ water, $7.7 \%$ protein, $2.4 \%$ soot, $0.45 \%$ natrium and $0.1 \%$ lipid.

We thought fish protein was denatured a little because this fish protein was come from the fish which was being heated. We used them with experiment for a new evaluation method. After heating, the fish protein would be brittle.

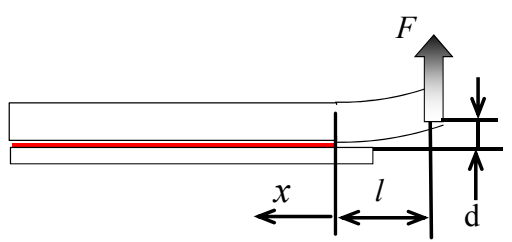

(a) Starting the experiment

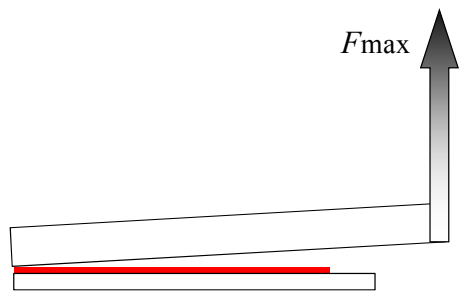

(b) After the separation

Fig. 2 The schematic of the peeling experiment

(a) Starting the experiment,

(b) After the separation 


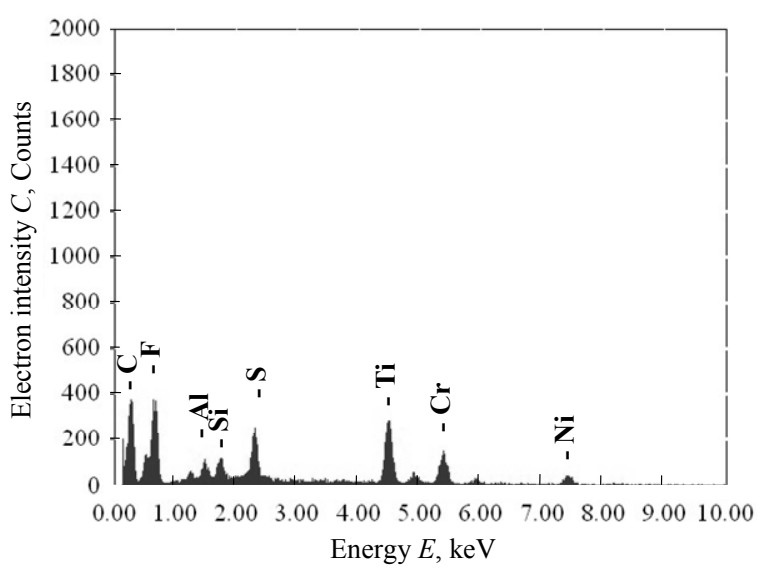

Fig. 3 The result of analysis of the fluorine resin (a) by EDX

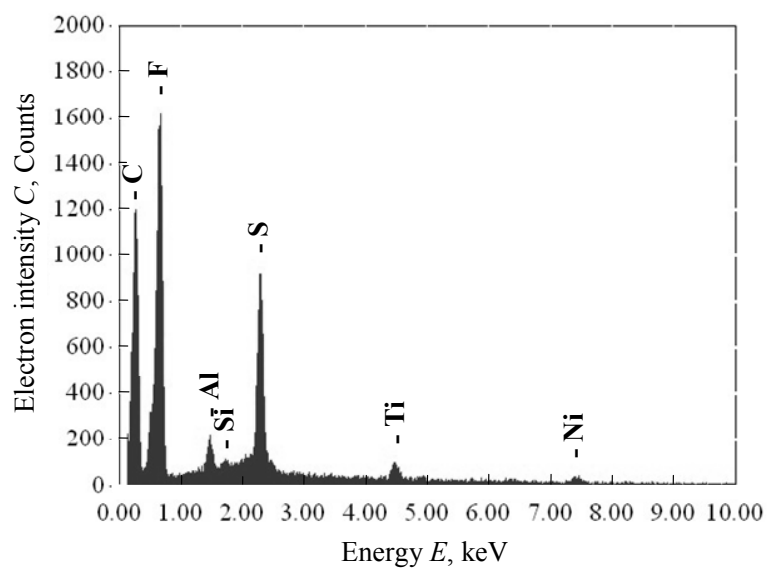

Fig. 4 The result of analysis of the fluorine resin (b) by EDX

\subsection{Specimen preparation}

We prepared some specimens for this peeling experiment. We thought we needed some grill rods which are used as the home gas cooker generally, $\mathrm{NiCr}$ coating grill rod and two kinds of the fluorine resin coating grill rods. Characteristics of $\mathrm{NiCr}$ coating grill rod are that it became adhesion to the fish from first but it is possible to clean for washing moderately. Characteristics of two kinds of the fluorine resin coating rods are that it don't become adhesion to the fish at first and if so, it was easy to clear by a few times. But we use these grill rods again and again, it became adhesion to fish strongly, and it was difficult to clean without washing strongly.

Here, we explain about some difference of two kinds of the fluorine resin grill rod specimens. We checked the ingredient material by energy dispersive X-ray spectrometry (called EDX), and showed these analysis results of the fluorine resin (a) as shown in Fig. 3 and the fluorine resin (b) as shown in Fig. 4. This electron accelerating voltage was $20 \mathrm{keV}$. The fluorine resin (b) had more ingredient of the fluorine resin against ingredient of carbon than the fluorine resin (a), and less ingredient of sulfur. The average surface roughness $R a$ of the fluorine resin (a) and (b) coating was $0.37 \mu \mathrm{m}$ and $0.72 \mu \mathrm{m}$, respectively.

\subsection{Experiment apparatus and procedure}

During grilling fish, the temperature in a grill equipment rises up to about $500{ }^{\circ} \mathrm{C}$, and the temperature at contact parts of the fish and the grill rods is about 150 ${ }^{\circ} \mathrm{C}$. So we decided to heat both specimens at $150{ }^{\circ} \mathrm{C}$ as experimental condition.

Figure 5 shows the experimental apparatus to measure the relationship between load and displacement during peeling off of a rod specimen against the denatured fish protein in a shallow groove on a plate specimen after heating. The rod specimen and the plate specimen were as shown in Fig. 1. The diameter and length of the rod specimen is $2.5 \mathrm{~mm}$ and $30 \mathrm{~mm}$, respectively. The radius and length of the groove of the plate specimen is $4 \mathrm{~mm}$ and $25 \mathrm{~mm}$. After pouring $0.3 \mu \mathrm{l}$ of the fish protein on center on the groove of the plate specimen, the rod specimen was put on the groove. Both specimens were taken on a cupper holder which had been already heated at $150{ }^{\circ} \mathrm{C}$ in an electric furnace and were heated all at $150{ }^{\circ} \mathrm{C}$ for 5 minutes.

Heating at $150{ }^{\circ} \mathrm{C}$ denatures the fish protein, and the rod specimen and the plate specimen adhere on interface with the fish protein. And one minute later, the both specimens on the cupper holder were set on the measurement apparatus of the peeling, and the plate specimen was fixed by some screws. And the rod specimen was pulled up as shown in Fig. 1 and Fig. 2. We could get the trace of the peeling force and the displacement.

After the peeling experiment, we observed the contact area by scanning electron microscope (called SEM). Figure 6 shows the contact area

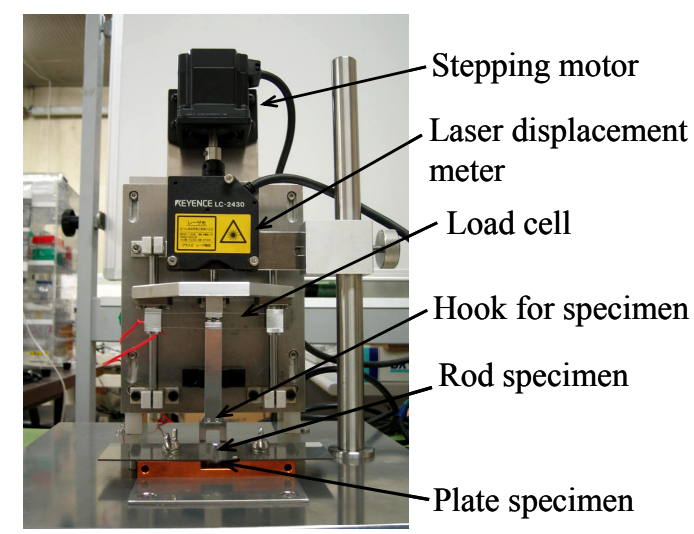

Fig. 5 The experimental apparatus for the peeling force $F$ and the displacement $d$ 


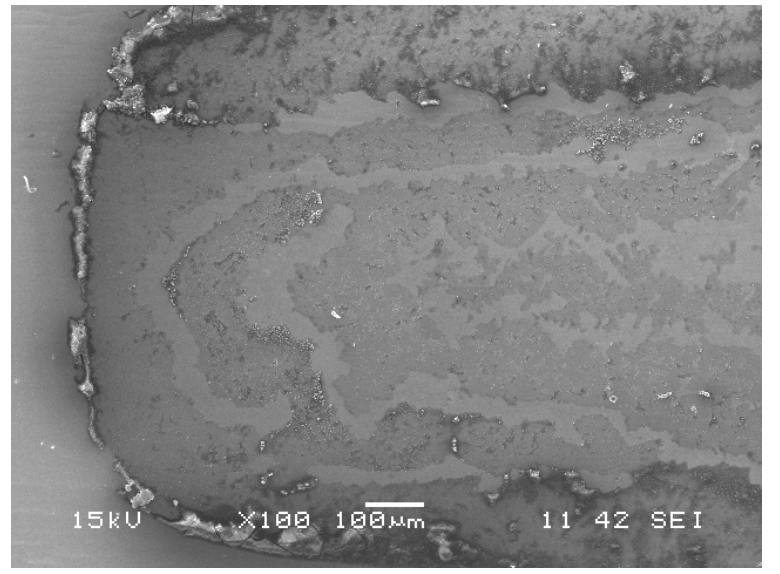

Fig. 6 The SEM image of the fracture area after the peeling experiment

\section{Results}

Figures 7-9 show some examples of the relationship between displacement $d$ of the end of rod specimen and peeling force $F$ between the rod specimen and the plate specimen with the fish protein after heating $150{ }^{\circ} \mathrm{C}$ for 5 minutes. Figures 7-9 shows the results for $\mathrm{NiCr}$ coating rod specimen, the fluorine resin (a) coating rod specimen, and the fluorine resin (b) coating rod specimen, respectively. It can be seen from these figures that peeling force increased with the increasing of the displacement up to the separation. Especially for Fig. 7 and Fig. 9, the peeling force was approximately proportional to the displacement up to the maximum force. In the case of Fig. 8, peeling force did not increase with the displacement proportionally. Because the fish protein would be brittle, so we thought the subsidiary fracture came inside the fish protein. Moreover, Fig. 10 shows the average peeling force $F$ by sixth times experimental results and its data spread bar.

Next, we calculated the value of the external applied work $W$ from the load-displacement curves as shown by Figs. 7-9. From Fig. 7 in the case of $\mathrm{NiCr}$ coating rod specimen, we calculated an approximate expression (3).

$$
y=1.24 \times 10^{-6}+9.64 \times 10^{-2} x-7.90 \times 10^{-4} x^{2}
$$

So the value of the external applied work $W$ for the fracture is calculated as $40.9 \times 10^{-6} \mathrm{~J}$ by integral calculation. Furthermore we estimated the contact area as $9.0 \mathrm{~mm}^{2}$ from the SEM observation as shown in Fig. 6. The interface fracture energy per unit contact area was calculated from both the external applied work $W$ for the fracture and the contact area. So the interface fracture energy per unit contact area at the interface between the fish protein and the $\mathrm{NiCr}$ coating rod is 4.5 $\mathrm{J} / \mathrm{m}^{2}$.

Similarly, we calculated the value of the applied external work $W$, the contact area and the interface fracture energy per unit contact area for the fluorine

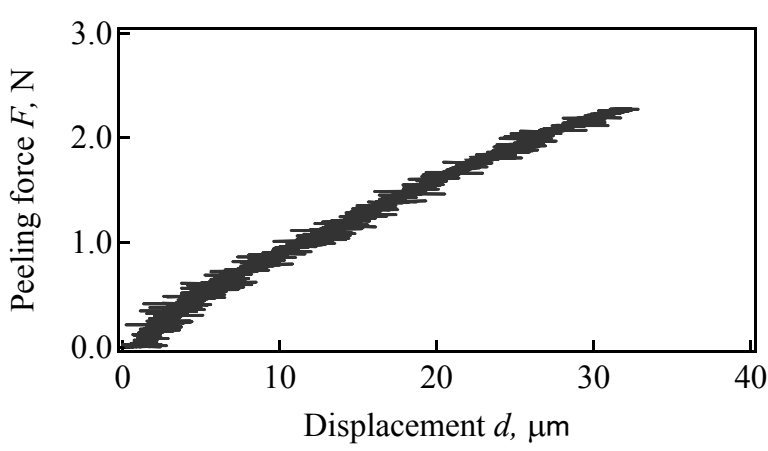

Fig. 7 The relationship between peeling force $F$ and displacement $d$ of the end of the $\mathrm{NiCr}$ grill rod during the peeling experiment

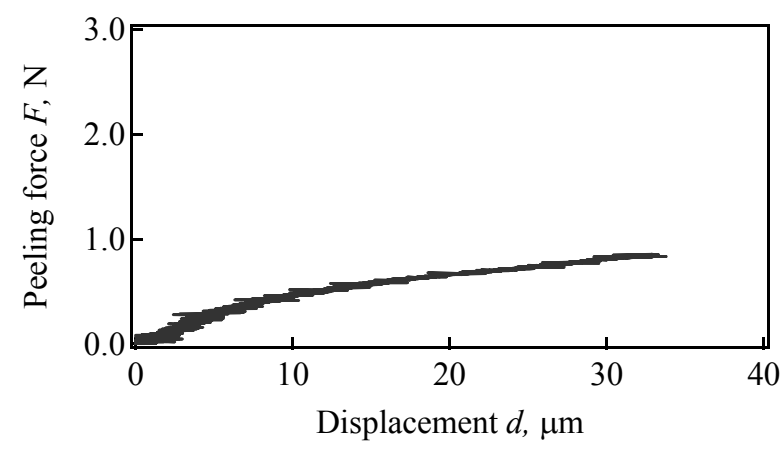

Fig. 8 The relationship between peeling force $F$ and displacement $d$ of the end of the fluorine resin (a) coating rod during the peeling experiment

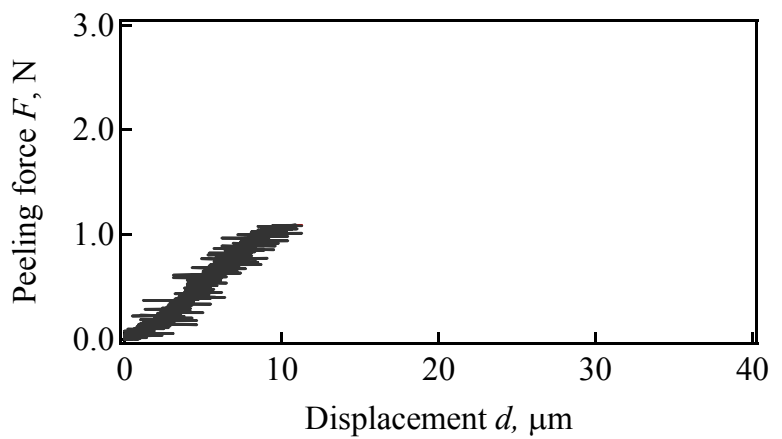

Fig. 9 The relationship between peeling force $F$ and displacement $d$ of the end of the fluorine resin (b) coating rod during the peeling experiment

resin (a) and (b) coating rods on the basis of Figs. 8-9. Table 1 shows the average peeling force, the average external work, and the average fracture energy per unit contact area for $\mathrm{NiCr}$ coating rod, the fluorine resin (a) and (b) coating rod.

\section{Discussions}

5.1. Advantage of the fracture energy comparing with maximum peeling force as the evaluation parameter

We focused on the load-displacement curves and calculated the external applied work $W$. And we 


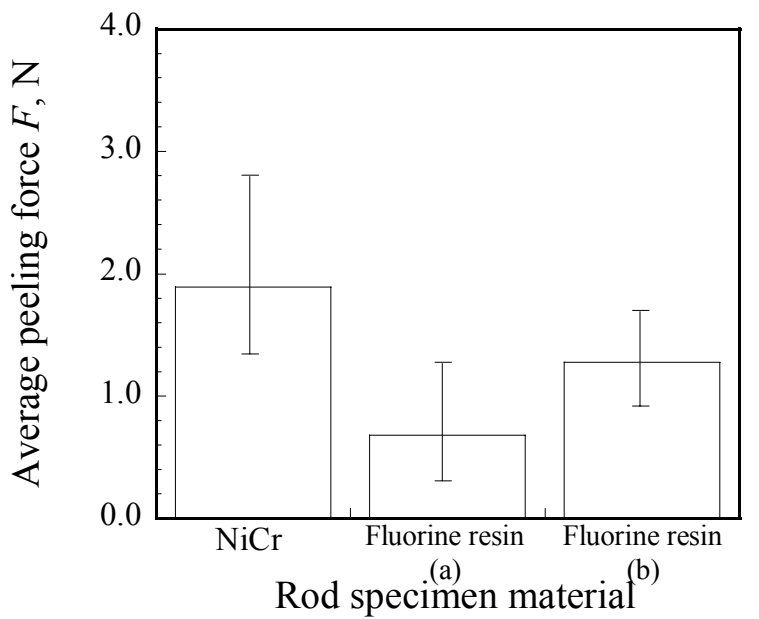

Fig. 10 The average peeling force $F$ and its data spread bar of three rod specimen material

measured the contact area $A$ by SEM and calculated the interface fracture energy $\Gamma$. Three graphs, Figs. 7-9, were much different in the view point of these peeling forces, displacements, and load-displacement curves. These differences had an effect on the external applied works and the interface fracture energy per unit the contact area. Table 1 shows the results of these average peeling force, external applied works and interface fracture energy per unit contact area. According to Table 1 , the low peeling force material is not low in the external applied works and fracture energy per unit the contact area. This reason includes a difference of the displacement until peeling off completely.

We consider that the value of the interface fracture energy $\Gamma$ was estimated on the basis of just simple assumption that external applied work $W$ was equal to the interface fracture energy $\Gamma$. So we got the value of the interface fracture energy $\Gamma$ by measuring the crack propagation process carefully.

5.2. Disadvantages of proposal method in the viewpoint of fracture position

The fracture positions are three possibilities to peel off as the followings between the fish protein and the rod specimen, the fish protein and the plate specimen, and inside the fish protein. The true purpose of this paper is to avoid the adhesion and not to decrease the peeling force. So we focus on the fracture at interface not the total fracture during peeling-off. However those unexpected phenomena as the deformation of fish protein should be occurred. We should subscribe the deformation of fish protein and grill rod specimens from the measured results. We should face this problem. Even when we grill fish in the equipment by gas usually, we do not know where may peel off between a fish and a grill rod clearly. But we think the fish protein is a factor of adhesion about fish and the grids of grill, because we observed the interface of peeling off carefully. So we guessed the most possible fracture position to peel off was between the fish protein and the rod specimen, and next possible was inside the
Table 1 The average peeling force, external applied work and interface fracture energy per unit area of three kinds of the rod specimen

\begin{tabular}{|c|c|c|c|}
\hline & $\begin{array}{l}\text { Average } \\
\text { peeling } \\
\text { force }[\mathrm{N}]\end{array}$ & $\begin{array}{l}\text { Average } \\
\text { external } \\
\text { work }[\mathrm{J}]\end{array}$ & $\begin{array}{l}\text { Average fracture } \\
\text { energy per unit } \\
\text { area }\left[\mathrm{J} / \mathrm{m}^{2}\right]\end{array}$ \\
\hline $\begin{array}{c}\text { NiCr } \\
\text { coating }\end{array}$ & 1.9 & 41.2 & 2.3 \\
\hline $\begin{array}{c}\text { Fluorine } \\
\text { resin (a) } \\
\text { coating }\end{array}$ & 0.67 & 17.9 & 1.1 \\
\hline $\begin{array}{c}\text { Fluorine } \\
\text { resin (b) } \\
\text { coating }\end{array}$ & 1.3 & 13.3 & 0.76 \\
\hline
\end{tabular}

fish protein. At first we discuss about the possibility if fracture position is inside the fish protein. Certainly, if the fracture point was inside the fish protein, we would not measure the accurate peeling force of interface.

And the fish protein was heated and denatured on the different specimen. But degree of denaturing for all of fish protein could be the same. Our measured energy could be the larger one in the fracture energy $W_{\mathrm{i}}$ of interface or deformation energy $W_{\mathrm{fp}}$ of denatured fish protein. Therefore if $W_{\mathrm{i}}$ is less than $W_{\mathrm{fp}}$, we can estimate the fracture energy $W_{\mathrm{i}}$ of interface. On the other hand, if $W_{\mathrm{i}}$ is larger than $W_{\mathrm{fp}}$, we can not estimate the fracture energy $W_{\mathrm{i}}$ of interface.

But, when the strain energy of denatured fish protein showed low value enough, this evaluation method for the metal grids of grill to the fish was applicable. Furthermore the advantages of this evaluation method were that it did measure the peeling force between the metal and fish directly, and it does not have an effect on the elastic coefficient of the fish, all kind of the fish. When we used the fish which exuded little fish protein, we could not apply to this evaluation method with the fish protein. So in this case, we used the fish meat instead of the fish protein and could measure the peeling force. But we have to think of the strain energy of each fish meat, it is so difficult to evaluate the fracture energy.

5.3. Reason why the fluorine resin (a) shows larger average external works than the fluorine resin (b)

After the experiment we observed the plate specimen, there was the trace of fish protein. So we observed the plate specimen to measure the contact area. Therefore we observed the plate specimens of two kinds of the fluorine resin (a) and (b). The average contact area of fish protein left on the plate specimen was that the fluorine resin (a) was $16.7 \mathrm{~mm}^{2}$ and the fluorine resin (b) was $17.9 \mathrm{~mm}^{2}$. We found that difference about the contact area between the fluorine resin (a) and the fluorine resin (b) by observing. And the trace length of fish protein on the plate specimen for the fluorine resin (a) was $21.0 \mathrm{~mm}$ and the fluorine resin (b) was $8.6 \mathrm{~mm}$. We found that in case of the fluorine resin (a), the traces of fish protein on the plate specimen were long and narrow, but in case of the 
fluorine resin (b), the traces of fish protein on the plate specimen were short and thick. These differences of way to expand have an effect on the displacement of the load-displacement curves and the contact area.

\section{Conclusion}

In order to develop the anti-stick surface of grid against a fish, new evaluation method of the peeling between fish protein and a rod specimen was proposed. Collected fish protein that was placed between a rod specimen and a plate specimen was heated up to $150{ }^{\circ} \mathrm{C}$ and denatured. And one minute later, the peeling force and displacement of the rod specimen and a plate specimen were measured to estimate the external applied work $W$ for the fracture at the interface. By using the value of contact area obtained with picture data, interface fracture energy $\Gamma$ per unit the contact area at the interface of the fish protein was estimated.
Furthermore, we measured to estimate adhesive properties of three kinds of grids of grill which we used usually $\mathrm{NiCr}$ coating specimen, the fluorine resin (a) specimen and the fluorine resin (b) specimen. And we calculated these peeling force, external applied work and interface fracture energy $\Gamma$ per unit the contact area.

\section{References}

[1] Griffith, A. A., "The Phenomena of Rupture and Flow in Solids," Philosophical Transactions of the Royal Society London, Series A, 221, 1920, 163-185.

[2] Pocius, A. V., "Adhesion and Adhesives Technology," The Nikkan Kogyo Shimbun, Tokyo, 1999, 15-42. (in Japanese) 